\title{
An Ensemble of Ensembles Approach to Author Attribution for Internet Relay Chat Forensics
}

\author{
SICONG SHAO, NSF Center for Cloud and Autonomic Computing, University of Arizona \\ CIHAN TUNC, Department of Computer Science and Engineering, University of North Texas \\ AMANY AI-SHAWI, National Center for Cybersecurity Technology, King Abdulaziz City \\ for Science and Technology \\ SALIM HARIRI, NSF Center for Cloud and Autonomic Computing, University of Arizona
}

\begin{abstract}
With the advances in Internet technologies and services, social media has been gained extreme popularity, especially because these technologies provide potential anonymity, which in turn harbors hacker discussion forums, underground markets, dark web, and so on. Internet relay chat (IRC) is a real-time communication protocol actively used by cybercriminals for hacking, cracking, and carding. Hence, it is particularly urgent to identify the authors of threat messages and malicious activities in IRC. Unfortunately, author identification studies in IRC remain as an underexplored area. In this research, we perform novel IRC text feature extraction methods and propose the first author attribution version of the deep forest (DF) model that is an ensemble of ensembles that utilizes the fusion of ensemble learning techniques. Our approach is supported by autonomic IRC monitoring. Experiments show that our approach is highly effective for author attribution and attains high accuracy even when the number of candidates is large while training data is limited.
\end{abstract}

CCS Concepts: - Security and privacy $\rightarrow$ Software and application security; • Computing methodologies $\rightarrow$ Machine learning; • Information systems $\rightarrow$ Data mining;

Additional Key Words and Phrases: Author attribution, ensemble learning, cybersecurity, social network analysis, internet relay chat (IRC)

\section{ACM Reference format:}

Sicong Shao, Cihan Tunc, Amany Al-Shawi, and Salim Hariri. 2020. An Ensemble of Ensembles Approach to Author Attribution for Internet Relay Chat Forensics. ACM Trans. Manage. Inf. Syst. 11, 4, Article 24 (October 2020), 25 pages.

https://doi.org/10.1145/3409455

This work is partly supported by the Air Force Office of Scientific Research (AFOSR) Dynamic Data-Driven Application Systems (DDDAS) award number FA9550-18-1-0427, National Science Foundation (NSF) research projects NSF-1624668 and NSF-1849113, (NSF) DUE-1303362 (Scholarship-for-Service), National Institute of Standards and Technology (NIST) 70NANB18H263, and Department of Energy/National Nuclear Security Administration under Award Number(s) DENA0003946.

Author's addresses: S. Shao and S. Hariri, are with the NSF Center for Cloud and Autonomic Computing, The University of Arizona, Tucson, AZ 85721; emails: \{sicongshao, hariri\}@email.arizona.edu; C. Tunc, is with the Department of Computer Science and Engineering, The University of North Texas, Denton, TX 76203; email: cihan.tunc@unt.edu; A. AI-Shawi, is with the National Center for Cybersecurity Technology, King Abdulaziz City for Science and Technology, Riyadh, 12354, Saudi Arabia; email: aalshawi@kacst.edu.sa.

Permission to make digital or hard copies of all or part of this work for personal or classroom use is granted without fee provided that copies are not made or distributed for profit or commercial advantage and that copies bear this notice and the full citation on the first page. Copyrights for components of this work owned by others than ACM must be honored. Abstracting with credit is permitted. To copy otherwise, or republish, to post on servers or to redistribute to lists, requires prior specific permission and/or a fee. Request permissions from permissions@acm.org.

(C) 2020 Association for Computing Machinery.

2158-656X/2020/10-ART24 \$15.00

https://doi.org/10.1145/3409455 


\section{INTRODUCTION}

There is rapid growth in the use of social networks to disseminate users' opinions and information immediately across the globe [Lau et al. 2011]. However, the downside of such social networks is that they have also been exploited by cybercriminals to create anonymous, fake, and illegal accounts [Wu et al. 2013; Samtani and Chen 2016] and to perform illegal activities including cyberattacks, hacking service trade, drug and weapon marketing, and money laundering [Abbasi et al. 2005; Benjamin and Chen 2015; Samtani et al. 2017], such as the Silk Road black market. Furthermore, these illegal cyber-activists can avoid detection using anonymous servers, spoofing, VPN, fake accounts, and so on. Hence, US homeland security and law enforcement agencies have launched multiple projects to prevent deceptive attacks and theft and to track hacker identities. Even though the anonymity methods have made the tracking extremely difficult, users leave some unintentional/unconscious traces, such as their messaging habits, which can be used to identify them. Therefore, author attribution through analyzing attacker messages is one of the most promising solutions, which can be formulated as follows: Given a set of the text of some candidates, assign a piece of text to the most likely candidate [Zheng et al. 2006; Segarra et al. 2015].

IRC is a real-time communication-based social network platform [Benjamin et al. 2016]. Even though IRC has been traditionally utilized for legitimate functions, it has also been the playground of anonymous users for malicious activities. Some channels even contain underground markets selling stolen credit card data, hacking services, security exploits, and so on [Benjamin et al. 2016; Samtani et al. 2016]. Therefore, identifying individual users through author attribution in IRC plays a key role in understanding cybercriminals' behaviors. For example, British police captured several targeted cybercriminals actively involved in "Operation Payback," the DDoS campaign launched by the Anonymous organization, through identifying the users in the AnonOps IRC channel [The Register 2012]. However, due to the anonymity provided by the IRC platform, a user can hide behind aliases that can easily be changed without registration. Further, a cybercriminal may leverage anonymity techniques for increasing the anonymity to frustrate network forensics. In such a case, the chat messages left on the IRC channels may be the only clue to identify users. Author attribution can discover identities through analysis of users' text. Therefore, author attribution in IRC can play a critical role in determining users.

Performing author attribution in IRC is a challenging task due to the following reasons: First, most of the previous work has focused on asynchronous computer-mediated communication (websites, forums, newsgroups, blogs, comments, etc.). In contrast, only a few studies have focused on author attribution in IRC, which is a synchronous medium. Second, in the malicious IRC channels, administrators remove/ban inactive users and bots and their IP addresses. This necessitates the development of intelligent and autonomous monitoring systems. Hence, our technique is supported by autonomic IRC monitoring. Third, in most author attribution studies, researchers have focused on author attribution among a limited number of authors up to 100 with significantly reduced accuracy and an increase in the number of authors [Abbasi et al. 2005; Zheng et al. 2006; Abbasi et al. 2008; Segarra et al. 2015]. For the IRC author attribution task, it is common that the number of candidates is larger with limited training data. Finally, cybercriminals in IRC channels may try to hide their identities to escape cybercrime investigation [Amuchi et al. 2012]. As a result, the studies of IRC author attribution are largely underexplored. This article attempts to tackle these challenges by providing a novel IRC author attribution forensic technique that can learn the holistic IRC writing feature sets through an ensemble of ensembles model.

The remainder of this article is structured as follows: In Section 2, we provide background and related work information about IRC, author attribution, and feature selection (FS) for text classification. Section 3 presents our novel IRC author attribution technique. Then, the experimental 
results are presented in Section 4. We provide further discussions and future works in Section 5. In Section 6, we conclude the article.

\section{RELATED WORK}

\subsection{Internet Relay Chat (IRC)}

IRC requires a server to provide networking through real-time text communication as publicly broadcasted messages in chat channels. The users can also point out the receiver, which highlights the messages to the receiver. This differs from the website behavior, because the IRC users cannot access previous messages [Benjamin and Chen 2014]. Thus, even though offline collection and batch processing would work for the websites/blogs, IRC requires real-time collection [Benjamin et al. 2016]. In this research, we create an autonomic IRC bot-based monitoring approach for comprehensive real-time recording and classification, as will be discussed in Section 3 .

\subsection{Author Attribution}

Author attribution is a task that can be formulated as assigning writing among a set of given authors [Zheng et al. 2006]. The origins of author attribution fall in the area of natural language processing (NLP). For example, Segarra et al. [2015] used function word adjacency networks to attribute text among 10 authors with an accuracy of $93.5 \%$ using $100 \mathrm{~K}$ words for each author. Zheng et al. [2006] developed an author attribution framework based on 270 writing-style features. They attributed up to 20 of the most active users who frequently posted messages in online newsgroup forums, with the best accuracy around 83\% when given 20 authors. Abbasi et al. [2008] proposed the Writeprints approach based on the extension framework of Zheng et al. [2006]. Instead of using the same features for all authors, they created individual feature sets for each author according to the individual's key features and executed author attribution experiments with 100 authors on three asynchronous mediums, including email, eBay comments, and Java Forum, with the best accuracy of $85.56 \%, 94.59 \%$, and $76.87 \%$. Tan and Tsai [2010] used a small feature set (50 features) from lexical and syntactic features to identify two selected authors' blog entries with $81.98 \%$ accuracy. Solorio et al. [2011] extracted writing features from web forum posts from 100 authors. Then, they combined unsupervised and supervised learning to perform author attribution given $5,10,20,50$, and 100 authors with an average number of words of $39,664,40,953,35,838,21,502$, and 11,322 , respectively. Their results achieved accuracy of $76.17 \%, 77.38 \%, 71.42 \%, 63.79 \%$, and $62.10 \%$, with 5, 10, 20, 50, and 100 authors. Unfortunately, author identification in IRC is very limited. Layton et al. [2012] attributed 50 users in the Ubuntu IRC channel and used inverse author frequency (IAF) weighting and re-centered local profile (RLP) methods to attribute authors. Their results achieved accuracies of over 55\%. Inches et al. [2013] performed author attribution in the krijn IRC dataset and the IRC-logs dataset, separately. The krijn IRC dataset focuses on the topic of the HTML5, and the topics of the IRC-logs dataset range from the programming language and operating system to database and hardware. By applying statistical models with chi-squared distance and Kullback-Leibler divergence, they achieved the best accuracy of $95 \%$, with 343 users in the krijn IRC dataset. However, their accuracy significantly reduced to $61 \%$ and 54\%, with 148 and 314 users in the IRC-logs dataset, respectively.

\subsection{Feature Selection for Author Attribution}

The dimensionality of authorship data is usually high (e.g., thousands of features are extracted to represent a single writing sample), where many redundant and irrelevant features may be included. Feature selection (FS) is an important procedure for reducing dimensionality, shortening training time, improving model performance, and gaining a better insight into the underlying process of 


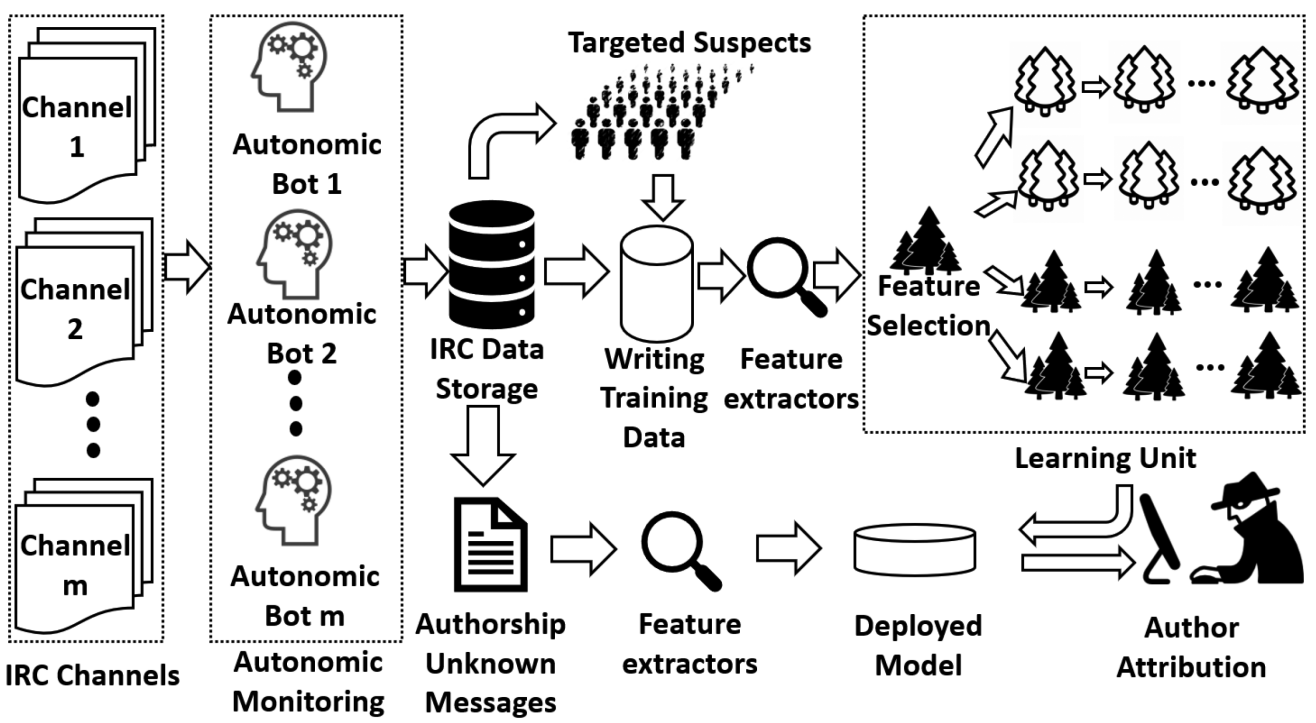

Fig. 1. The architecture of the IRC author attribution approach.

data generation. Therefore, FS is highly useful for author attribution tasks. Mukras et al. [2007] used information gain-based FS methods for machine learning-based text categorization. Alrabaee et al. [2014] measured the degree of correlation to rank and select features for authorship analysis. Maruf et al. [2016] improved text classification performance with random forest (RF)-based FS, compared with other FS methods, including information gain, odds ratio, and information gainprincipal component analysis.

\section{IRC-BASED AUTHOR ATTRIBUTION APPROACH}

Figure 1 shows our IRC author attribution approach architecture. First, using our autonomic IRC bots deployed in various IRC channels, mainly about hacking and cracking activities, we monitor the IRC channels and users' messages and behaviors. The IRC messages of author candidates are extracted to perform two major steps for author attribution: feature extraction and author attribution deep forest (AADF) construction. The AADF construction contains two phases: FS phase and attribution model learning phase. After that, through author attribution model, the extracted unknown authorship messages represented by selected features can be attributed to the author candidates.

\subsection{Autonomic IRC Bot}

We developed the autonomic IRC bot to collect real-time communication from IRC channels. Before describing the detail of our IRC bot, we briefly discuss the bot architecture at a high level. As shown in Figure 2, our autonomic bot consists of the modules of conversation, real-time data collection, real-time threat message detection, and self-replication. The conversation module is used to provide chat capability through IBM Watson Assistant service [IBM Watson Assistant 2018]. The real-time data collection module is designed to monitor the IRC channel and perform data collection. Furthermore, the real-time threat message detection module can classify the threat level of IRC messages through deep learning. The classified IRC messages can be used for hacker data analysis. The self-replication module is designed to create new autonomic bots. For each IRC channel, our bots monitor the messages and transform the collected data to structured data with 


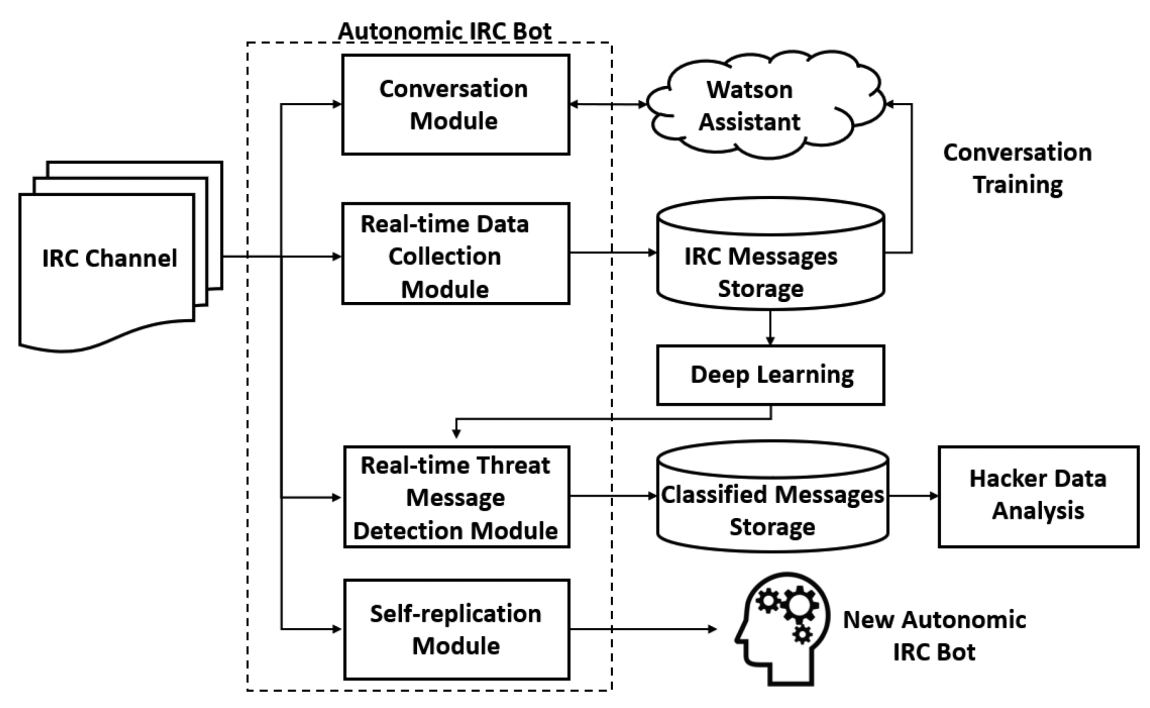

Fig. 2. The architecture of autonomic IRC bot.

the following format: Threat level + User nickname + Chat content + Date + Time. Thus, our autonomic bot is built with robust continuous monitoring, comprehensive information collection, preprocessing, and classification in real-time.

Concretely, we develop the conversation model to support the real-time data collection module, since the IRC channel operators ban users and even block their IP if a user is identified as an inactive user or a bot in some malicious channels. Our IRC bots leverage the intelligent conversation capability that responds to chat like a real user to prevent identification by operators. Using Watson Assistance [Bernard et al. 2018; Kratzwald and Feuerriegel 2019], the bots understand the chat inputs and respond to IRC users by mimicking human conversations as follows: (1) We create a workspace that is a container for dialog flow and training data. (2) The workspace is configured using training data collected from the chat messages. We transform the chat messages into intent, entity, and dialog contents in the workspace for training. The intent is the IRC user's anticipated goal, e.g., Distributed Denial of Service (DDoS) [Li et al. 2015]. For each intent, we add training samples reflecting what IRC users might ask for the information they need; e.g., "Which tool is available to perform a DDoS attack?" The entity represents a term, an object, or a data type that provides context for an intent. The dialog is a branching conversation flow that defines intents and entities. Through the use of the dialog tool incorporating IRC user's intents and entities, we can add a branch to process every intent that we want the bot to respond to. (3) After the creation of the chat model for the monitored IRC channels, we integrate IBM Watson Assistance into the conversation module of the bot.

Many hacker channels publish self-signed certificates and prevent non-SSL connections. Hence, self-signed certificate creation and port number are added to the real-time data collection module of our autonomic IRC bot. Also, cybercriminals can create temporary channels to disseminate hacking knowledge or share malicious tools, and even organize cyberattacks. For example, on December 6, 2010, the AnonOps server users started using a temporary channel called \#operationpayback, which had been quiet for months [Sauter 2014]. In this channel, the cyber attackers discussed their motivation and plan. They then started launching DDoS attacks against the websites of the Swedish Prosecution Authority, everyDnS, Senator Joseph Lieberman, and so on, resulting in downtime for these websites [Sauter 2014]. Hence, to continuously track such activities, 


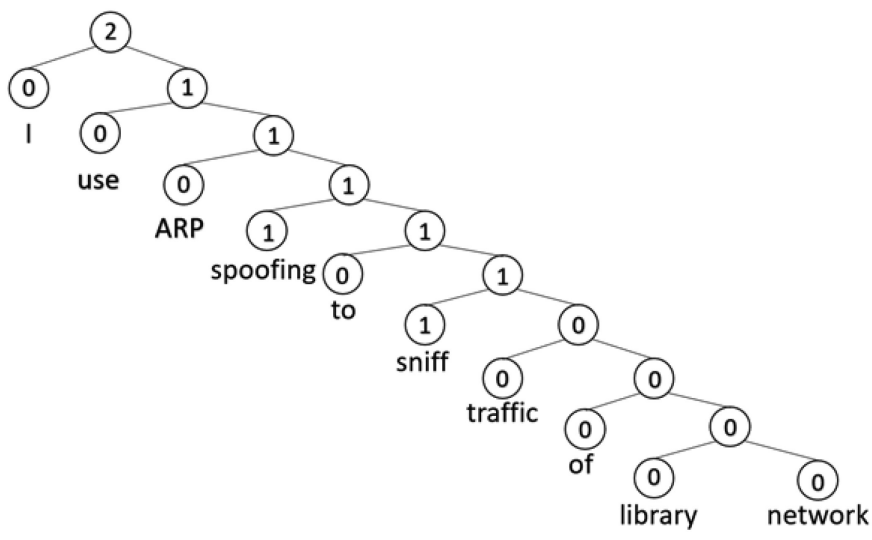

Fig. 3. An example of our labeling rule, from normal to high $(0,1,2)$ at every node of the threat tree construction.

a self-replication module is developed to allow the parent bot to generate a new (child) bot that inherits all the parent bot's capabilities. The recursive neural tensor network (RNTN) has been proven to be powerful for sentiment analysis [Socher et al. 2013]. The RNTN deep learning model represents words and phrases as D-dimensional vectors through recursively performing tensorbased composition functions to the parse tree. Words of a sentence are represented as numeric vectors and combined by tensor-based composition functions to form parent vectors in a bottomup way. Using RNTN, the autonomic IRC bot can automatically distinguish between normal and threat chat messages and identify their threat level. The recursive deep learning model can classify each IRC message into three defined levels: Normal (Score 0, for no malicious contents), Warning (Score 1, indicating a potential risk because of the use of hacking terms), and High (Score 2, denoting the sender appears to have some malicious behaviors or intentions). The labeling rule for the current node score that depends on the threat level of the whole phrase that the current node dominates under the parse of the threat tree is shown in Figure 3.

\subsection{Feature Extraction}

Profile-based author attribution methods and instance-based author attribution methods are two approaches to perform author attribution. The profile-based approach concatenates the available texts of a certain author to one single text file. Therefore, each author has only one big author profile file [Stamatatos 2009]. The training process of the profile-based approach is simple, since the training phase comprises feature extraction from candidate profiles. Then, the author attribution model uses a distance function to compute the most likely author. The majority of modern author attribution approaches consider each text sample as an instance of the known candidate. Unlike the profile-based approach, which has only one long training sample for each suspect, a long text for a certain candidate should be segmented into multiple training samples, probably of equal length in instance-based approaches [Stamatatos 2009]. In this research, we perform non-overlapped segment method to each author's whole IRC messages (to create multiple samples), with $1 \mathrm{~K}$ words per sample. Then each sample is used to extract features from the feature set extractors. Table 1 summarizes the feature sets used in this study.

3.2.1 Letters and Numbers and Special Characters and Punctuations. This feature set is one of the most commonly used features in previous research [Zheng et al. 2006; Abbasi et al. 2008]. For each sample, we count the occurrences of 52 letters (case-sensitive), 10 numbers, and all the 
Table 1. Description of Feature Sets

\begin{tabular}{|c|c|c|}
\hline Feature Set & Description & $\begin{array}{l}\text { The } \\
\text { Number of } \\
\text { Features }\end{array}$ \\
\hline $\begin{array}{l}\text { F1: Letters, numbers, } \\
\text { special characters, } \\
\text { punctuations }\end{array}$ & $\begin{array}{l}\text { Frequency of letters, numbers, special characters, } \\
\text { and punctuations }\end{array}$ & 94 \\
\hline F2: Character n-grams & $\begin{array}{l}\text { Frequency of the most } 500 \text { frequently ranked } \\
\text { character } 2 \text {-grams and the most } 1,000 \text { frequently } \\
\text { ranked character } 3 \text {-grams }\end{array}$ & 1,500 \\
\hline F3: Word n-grams & $\begin{array}{l}\text { Frequency of occurrence of the most } 500 \\
\text { frequently ranked word } 2 \text {-grams and the most } \\
1,000 \text { frequently ranked word } 3 \text {-grams }\end{array}$ & 1,500 \\
\hline F4: Stop-words & Frequency of stop-words & 543 \\
\hline $\begin{array}{l}\text { F5: Cybersecurity } \\
\text { knowledge and attitude }\end{array}$ & $\begin{array}{l}\text { Frequency of using NIST key information } \\
\text { security terms }\end{array}$ & 6,702 \\
\hline F6: Abbreviation & Frequency of IRC abbreviations & 46 \\
\hline F7: Emoticons & Frequency of IRC emoticons & 37 \\
\hline F8: Activity Behaviors & Amount of IRC messages in each half-hour & 48 \\
\hline F9: Personality Insights & Big Five Model, Needs model, Values Model & 52 \\
\hline $\begin{array}{l}\text { F10: Public pair } \\
\text { conversation network }\end{array}$ & $\begin{array}{l}\text { Frequency of sending public one-to-one messages } \\
\text { to a candidate }\end{array}$ & varies \\
\hline
\end{tabular}

21 special characters used in the study of Zheng et al. [2006]. Since IRC messages might be informal text, the frequency of using punctuation features might also be reliable in IRC author identification. We adopted 11 punctuations (“.”, “,”, “?”, “!”, “’”, “””, “:”, “,”, “...”, “-”, “-”) in our research.

3.2.2 Character $\mathrm{N}$-grams. The character $\mathrm{n}$-grams feature set is commonly leveraged by authorship analysis tasks [Abbasi et al. 2008; Layton et al. 2013]. This feature set contains information such as writing behaviors related to capitalization, space, morphological variants of words, typos, and so on. We extracted the most frequent occurrence of character n-grams for feature creation with a frequency ranked cutoff point. Consider the fact that there are cases when the total number of underlying character n-grams types is small due to very limited text samples or frequent repetitive text, which is possible in social media forensics. To make sure this feature extractor still can work in such cases, we do not consider the low-ranking character n-grams. Therefore, we simply limit the size of this feature set to $\mathrm{n} \in\{2,3\}$ with a frequency ranked cutoff point of 500 for character 2-grams and 1,000 for character 3-grams to make this feature extractor more general. We use more character 3-grams features than character 2-grams due to the fact that the dimensionality of character 3-grams features is much higher than character 2-grams features.

3.2.3 Word N-grams. Users might have the habit of using some phrases constructed by two or more sequential words. Thus, word n-grams features have been effectively used for author attribution [Stamatatos 2009]. We conjecture that word n-grams would also be useful for the identification of IRC users. We use the bag-of-words model to represent the feature frequency [Stamatatos 2009]. Apache Lucene library [Białecki et al. 2012; Williams et al .2014] is used to filter word n-grams. For the same reason as the character n-gram feature set, a ranked cutoff point is set where the 500 most frequently ranked word 2-grams and 1,000 most frequently ranked word 3-grams are 
selected. This way, each sample contains 500 most frequently ranked of word 2-grams features and 1,000 most frequently ranked of word 3-grams features. Compared to the character n-grams feature set, the word n-grams feature set can capture longer sequential writing information, since one word generally contains multiple characters. Another difference is that word-level n-grams are able to extract more semantic meaningful knowledge from IRC messages, while the character-level $\mathrm{n}$-grams can capture uncommon features such as typos and morphological variants.

3.2.4 IRC Stop-words. We explore the stop-words feature set, since stop-words typically contain most of the function words (function words determine the syntactical form of a sentence). Stop-words are generally the most common words in a language; there is no single universal list of stop words used by all NLP tools, and any set of words can be selected as the stop-words for a given purpose [Rajaraman and Ullman 2011]. They are generally avoided by most search engines due to the high appearance frequency. However, in IRC-based author attribution, we conjecture that stop-words are suitable for IRC authorship analysis, since it could provide meaningful information about the IRC users, because individuals have their own ways of organizing stop-words. We use the stop-words listed on the website of the work by Sedgewick and Wayne [2018], which introduces a stop-words list. We measured the frequency of all the IRC stop-words in each user's writing sample to distinguish their characteristics.

3.2.5 IRC Cybersecurity Knowledge and Attitude. The users in an IRC channel have different levels of knowledge. For example, some are cybersecurity novices, but some are sophisticated hackers with advanced cybersecurity techniques. Similarly, their interest, involvement in activities, and so on, will differ as well. Therefore, the information security terms can be used to describe the cybersecurity knowledge, the interesting topic, and the activities of monitored individuals. Motivated by helping the user understand information security terminology, NIST has created a repository of key information security terms and definitions in their official webpage of the Computer Security Resource Center [NIST 2018], which is regularly updated. We extracted 6,702 terms from this glossary and used the occurrence frequency of each term.

3.2.6 IRC Abbreviation. Some users include abbreviations such as "afk" (away from the keyboard), "brb" (be right back), and so on, and we have used 46 common abbreviations listed in an IRC beginner website [IRC Abbreviations 2018], which introduce the commonly used abbreviations (see Appendix for the used abbreviations) in IRC channels to represent the user's behavior of typing abbreviations. Considering the relatively casual environment of IRC, the users' writing styles include abbreviations profoundly.

3.2.7 IRC Emoticons. The informal nature of IRC creates a need to express a user's emotions in a textual way; hence, we adopted 37 IRC text emoticon features (see Appendix for the used emoticons) from an IRC tutorial website [IRC Emoticons 2018]. Thus, the author can be identified by measuring the IRC emoticons feature set.

3.2.8 IRC Activity Behaviors. The activity behaviors of IRC users are impacted by many factors, such as living time-zone. We measure users' activity behaviors by the number of messages posted in different time slots to unveil clues about users. We create time interval features, where a day is defined using 48 equal half-hour intervals. The main reason for choosing this time interval is that our observations show that the activities of IRC users seem to be consistent during half-hour.

3.2.9 IRC Personality Insights. The users' personality characteristics are unique from individual to individual and relatively stable [Cobb-Clark and Schurer 2012]. Based on how IRC users communicate with others, personality characteristics influence most of the user's activities and behaviors. Moreover, personality, which is expressed in their messages, also influences the way 
IRC users make decisions, including cyberattack types and hacking techniques selection, attack and crime motivation, hacking organization membership, and malicious tools being developed. Using IBM's Personality Insights services [IBM Watson Personality Insights 2018], we can successfully analyze individual authors' IRC messages and intrinsic personality characteristics to create their personality profiles. In this work, we have used English only, but the application to the other languages should be straightforward. The Personality Insights service provides an individual's characteristics in JSON format based on three models: Big Five, Needs, and Values. Big Five model contains the following five primary dimensions: agreeableness, conscientiousness, extraversion, emotional range, and openness. Each of these primary dimensions includes six facet features to further distinguish a user. Needs model contains 12 features, and Values model includes five features. We selected all facet features and primary features to represent the personality of the users (see the used features in Figure 5).

3.2.10 IRC Public Pair Conversation Network. In IRC channels, users congregate with conversation networks based on their thread knowledge, capabilities, criminal interests, friendship, and so on. While some users are interested in and experienced in carding crimes, some are specialized in developing vulnerability detection tools, exploitation techniques, and some focus on disseminating hacking tools and recruiting new members. Hence, by studying the pair conversation network of IRC channels, we can build representation of each candidate's pair conversation networks. For public IRC messages, users can point out the receiver's nickname that highlights to whom the messages are being sent. For the candidate sample, we, respectively, count the number of each candidate's highlighted messages from this certain candidate sample. Therefore, each sample will generate the features that may characterize their unique pair conversation networks in the IRC channel.

\subsection{Self-adaptive Author Attribution Deep Forest}

$\mathrm{DF}$ is a tree-based ensemble of ensembles framework that can operate well even with relatively less data [Zhou and Feng 2017]. By using a fusion of ensemble techniques, DF allows layer-by-layer processing, representation learning through the forest, in-model feature transformation, sufficient model complexity, and self-adaptive structure. For IRC author attribution, we propose the first DFbased author attribution approach called author attribution deep forest (AADF). Figure 4 shows our self-adaptive AADF architecture. The original version of DF consists of a cascade forest structure (CFS) module and another module called multi-grained scanning. We replace the multi-grained scanning module with our FS unit, because the multi-grained scanning module is used for the analysis of spatial and sequence relationships among raw features. This is unnecessary for our extracted IRC writing style features, since they do not convey spatial or sequential relationships.

FS is an important step in the author attribution machine learning pipeline. The dimensionality of authorship data is usually high (e.g., thousands of features, such as character n-grams, word $\mathrm{n}$-grams, and stop-words, are extracted to represent a single writing sample), whereas irrelevant features may be included resulting in time-consuming and resource-consuming. To reduce this problem, in AADF, the estimates of feature importance can be calculated, and FS can be performed using the tree ensemble model. Thus, feature dimensionality can be significantly reduced while the attribution performance is maintained or even improved.

The most interesting part of DF from the view of author attribution is the CFS, which is a boosting-related and stacking-related procedure. To enhance diversity (highly suggested for ensemble learning), our version uses two types of base tree ensemble models, which are random forest (RF) and extremely randomized trees (ERT). The RF model consists of an ensemble of decision trees on various sub-samples and uses averaging to improve the performance. Also, the best split 


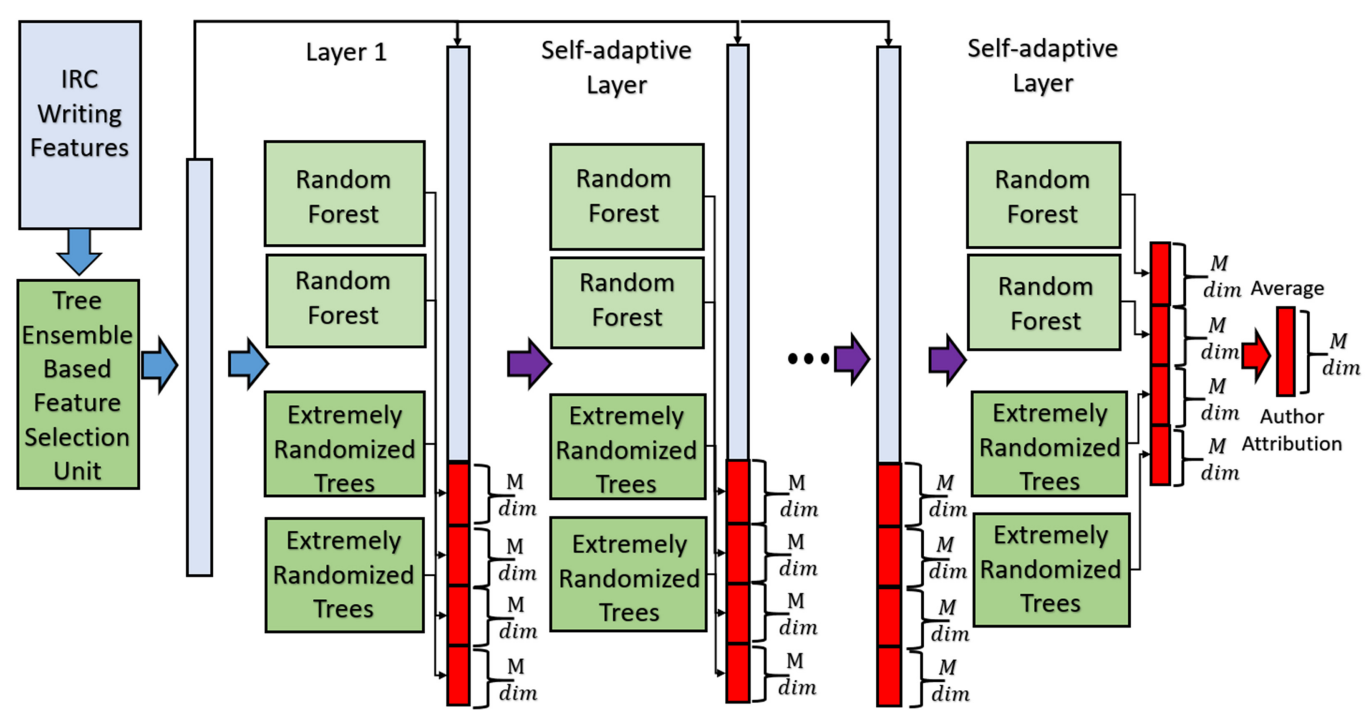

Fig. 4. Self-adaptive AADF architecture example with base tree ensemble models in each layer and $\boldsymbol{M}$ candidates. Thus, each base tree ensemble model will produce an $\boldsymbol{M}$-dimensional class vector, and each layer will output a $(\boldsymbol{P}+\boldsymbol{Q}) \cdot \boldsymbol{M}$ dimensional class vector as augmented features. Here, suppose there are two RF models and two ERT models at each layer of AADF. The augmented features are then concatenated with the output vector of the feature selection unit as the new representation and used as an input vector to each base tree ensemble model of the next layer.

is determined from a random subset of total features when splitting each node during decision tree construction. The purpose of these two randomnesses is to increase the variance of individual decision trees and, therefore, to decrease the variance of the forest model by combining diverse trees [Breiman 2001]. The ERT model is a tree-based ensemble model for supervised learning [Geurts et al. 2006]. Compared with RF, it is further randomness in the way splits are computed. Instead of searching for the most discriminative thresholds for random subset of candidate features, ERT draws thresholds at random for each candidate feature. Then the best of these random thresholds is used as the splitting rule. Therefore, the variance of the ERT model is reduced while its bias is increased.

Let a given training dataset $X=\left\{x_{i}, y_{i}\right\}\left(x_{i} \in \mathbb{R}^{d}, y_{i} \in Y,|Y|=M, i=1,2,3, \ldots, n\right)$ be drawn independently and identically distributed (i.i.d) from distribution $\mathcal{D}$, where $d$ is the total number of features after feature extraction, $d^{\prime}$ is the total number of features after the processing of FS unit, and $M$ is the total number of classes. Then, the input of CFS is $X_{0} \in \mathbb{R}^{n \times d^{\prime}}$. Each layer has $P$ total RF models and $Q$ total ERT models. Each base tree ensemble model (RF and ERT) can generate a class vector by averaging the class prediction probability across all the trees of that particular base tree ensemble model. Hence, a $Z$-dimensional class vector is generated as augmented features, where $Z$ is the product of $(P+Q)$ and $M$. Let $l$ be the index of each layer, where $l=1,2, \ldots, h$ and $h$ is the total number of the layer in the CFS. Except for the layer 1, we call other layers as self-adaptive layers, since the number of self-adaptive layers can be automatically determined during the training phase. Except for the final layer, the class vector of the layer $l$ is used as augmented features that are then concatenated with the input $X_{0}$ as the new representation and used as input vector to each base tree ensemble model in the next layer. The input data 
$X_{l}$ of the layer $l+1$ is given as:

$$
\begin{aligned}
& V_{l}=\left\{v_{l, R F_{1}}, v_{l, R F_{2}}, \ldots, v_{l, R F_{p}} ; v_{l, E R T_{1}}, v_{l, E R T_{2}}, \ldots, v_{l, E R T_{q}}\right\} \in \mathbb{R}^{n \times Z} \\
& X_{l}=\left\{V_{l} ; X_{o}\right\} \in \mathbb{R}^{n \times Z^{\prime}},
\end{aligned}
$$

where $v_{l, R F_{p}}$ and $v_{l, E R T_{q}}$ are class vectors generated by RF with index $p$ and ERT with index of $q$ in layer $l$, and $Z^{\prime}=Z+d^{\prime}$, and $V_{l}$ is the augmented feature vector generated by layer $l$. The class vector generated by each tree ensemble model is produced by $k$-fold cross-validation. More specifically, each sample will be used as a training sample for $k-1$ times, generating $k-1$ class vectors, that are then averaged to generate the class vector, which will be used as augmented features for the next layer. After growing one more self-adaptive layer, the prediction accuracy of the whole cascade structure is estimated by the validation set, and the growth of the cascade structure is automatically terminated if the class prediction accuracy does not increase any further. Hence the total number of layers can be self-determined in a data-dependent way, making the complexity of AADF self-adaptive with the authorship data. Then, the class vector in the final layer $h$ is given as:

$$
V_{h}=\frac{\sum_{\mathrm{p}=1}^{P} v_{h, R F_{p}}+\sum_{\mathrm{q}=1}^{\mathrm{Q}} v_{h, E R T_{q}}}{P+Q} \in \mathbb{R}^{\mathrm{n} \times \mathrm{M}}
$$

Then each sample is attributed to the class with the highest prediction probability in the class vector of the final layer. One of the advantages of the deep-forest-based model compared with most machine learning models is that it is quite robust to the hyper-parameters setting. Even across a broad range of tasks, the same setting of CFS of DF can achieve excellent performance [Zhou and Feng 2017]. In this research, to emphasize that the hyper-parameter setting of AADF is also simple and robust in author attribution tasks, we use the same setting of CFS across all the experiments of AADF. For simplicity, in AADF, suppose that two RF models and two ERT models are used in the CFS. Each RF contains 500 trees, and each ERT has 650 trees. However, please note that a careful hyper-parameter tuning and more computational resources may obtain better author attribution performance, though we have not tried more forests and trees due to the limit of computational resources.

The cascade procedure of AADF is related to boosting, since it is able to automatically decide the number of classifiers in the ensemble. Using the output of one level of classifiers as the input to the next level of classifiers is related to stacking. However, traditional stacking is easy to overfit with more than two levels and can hardly enable a deep model [Zhou and Feng 2017]. The cascade forest structure aims to solve the problem by concatenating its input $X_{0}$ and augmented feature vector $V_{l}$ generated by layer $l$ as the new input for self-adaptive layer $l+1$.

\section{EXPERIMENTS AND RESULTS}

\subsection{Corpus}

To evaluate our approach, we performed author attribution on highly heterogeneous IRC channels (shown in Table 2). The monitored channels are as follows:

- \#anonops: Controlled by the infamous Anonymous hacking organization, which is involved in many cybercrimes [Benjamin et al. 2016; Bernard et al. 2018].

- \#2600: A highly active community with hacker magazines and monthly hacker meetings [Shao et al. 2018].

- \#darkscience and \#hak5: Hacker communities sharing the interest in security.

- \#computer: A discussion channel for the Underworld server for cybersecurity and cybercrimes.

- \#thepiratebay.org: Copyright infringement and participating in the swarm for illegal files. 
Table 2. Total Number of Collected Messages in the Monitored IRC Cybersecurity Channels

\begin{tabular}{lccc}
\hline Server & Channel & \#Messages & Collection Date Range \\
\hline irc.anonops.com & \#anonops & $1,326,580$ & $8 / 15 / 17-9 / 20 / 18$ \\
irc.2600.net & \#2600 & 785,849 & $4 / 01 / 17-9 / 20 / 18$ \\
irc.darkscience.net & \#darkscience & 165,896 & $10 / 11 / 17-9 / 20 / 18$ \\
irc.darkscience.net & \#hak5 & 128,389 & $4 / 01 / 17-9 / 20 / 18$ \\
irc.underworld.no & \#computer & 361,320 & $9 / 13 / 17-9 / 20 / 18$ \\
irc.underworld.no & \#thepiratebay.org & 81,095 & $3 / 13 / 18-9 / 20 / 18$ \\
irc.underworld.no & \#trading & 75,996 & $11 / 28 / 17-9 / 20 / 18$ \\
irc.freenode.net & \#networking & 338,493 & $9 / 06 / 17-9 / 14 / 18$ \\
irc.freenode.net & \#security & 289,007 & $4 / 13 / 17-9 / 20 / 18$ \\
irc.freenode.net & \#bash & 266,098 & $9 / 06 / 17-9 / 20 / 18$ \\
irc.freenode.net & \#bitcoin & 164,663 & $9 / 13 / 17-9 / 20 / 18$ \\
irc.undernet.net & \#undernet & 310,723 & $12 / 05 / 17-9 / 20 / 18$ \\
\hline
\end{tabular}

- \#trading: An Underworld channel with the topic of black markets.

- \#security, \#networking, and \#bash: Three popular channels involving cybersecurity and computer.

- \#bitcoin: Frequent discussing of cryptocurrency usage in black markets and illegal trading.

- \#undernet: Hacking and cracking on the Undernet server.

In author attribution tasks, many studies tend to use over 10,000 words per author for experiments, which is treated to be a reliable minimum [Luyckx and Daelemans 2008]. As the words per text sample, 1,000 words is regarded to be a suitable choice [Hirst and Feiguina 2007]. Hence, we used ten 1,000-word text samples of each user for experiments. As a result, in the monitored 12 IRC channels, the different users who send more than 10,000 words are extracted to experiment with author attribution, resulting in 441 different users to experiment. Each user's whole messages are concatenated and then partitioned to create non-overlapping samples with equal 1,000 words (the remaining messages that are less than 1,000 words are discarded). Next, we apply random undersampling to the corpus [Liu et al. 2009]. We perform random undersampling to the majority by randomly removing samples from that population until the minority class becomes some specified ratio of the majority class [Imbalanced-learn API 2019]. We set the ratio of the biggest class to the smallest class that is $1: 1$ to balance the class. Therefore, after random undersampling, each candidate has equal ten 1,000-word samples in the dataset for experiments. Then, the total number of samples is 4,410 with 441 IRC users in the experimental dataset. We named this dataset 1.

To enhance the diversity of our experiments, the IRC public datasets provided by AZSecure-data project [Samtani et al. 2016; Du and Zhang 2018] are also used, which include AnonOps, Ed, and Hacker IRC channels. Hacker channel is known for facilitating the activities of the Anonymous hacktivist group. Ed channel has not been originally intended for hacker discussion, but a large number of hackers and hacktivists use it to communicate and share knowledge, due to its popularity and anonymity [Du and Zhang 2018]. All the channels' data is collected from September 2016 to May 2018. For the AnonOps channel, the data after August 15th, 2017, is dropped to avoid overlap with our collected AnonOps channel data in dataset 1. For the Hacker and Ed channels, all the data is considered. Then, we use the same strategy used for creating dataset 1 to create dataset 2 , resulting in 185 different users with ten 1,000-word samples for each user. The descriptions of dataset 1 and dataset 2 are presented in Table 3. 
Table 3. Description of the Experimental Datasets

\begin{tabular}{lccccc}
\hline Dataset & Authors & Features & Samples per Authors & Words per Samples & Samples \\
\hline Dataset 1 & 441 & 10,963 & 10 & 1,000 & 4,410 \\
Dataset 2 & 185 & 10,707 & 10 & 1,000 & 1,850 \\
\hline
\end{tabular}

\subsection{Results}

In this section, we validate the effectiveness of AADF approach and compare AADF with competing approaches. We first investigate the attribution performance of different approaches without FS unit. After that, we compare AADF to other approaches with tree ensemble-based FS units.

We use all the feature set extractors described in Section 3.2 to perform feature extraction. The min-max normalization [Scikit-Learn MinMaxScaler 2019] is applied for feature scaling. We compare the 10-fold cross-validation results of state-of-the-art author attribution classification models to the AADF approach. The support vector machine (SVM), naive Bayes (NB), and random forest $(\mathrm{RF})$ are considered as state-of-the-art author attribution classification models [Altakrori et al. 2018]. Artificial neural network (ANN) is also used for comparison due to that it is also high success in the author attribution domain [Baron 2016] as well as on various tasks. For SVM, we use the linear kernel, radial basis function kernel, polynomial kernel, and sigmoid kernel, respectively. For NB, we use the multinomial NB (MNB) classifier, because it is suitable for text classification [Manning et al. 2008]. The DF's base ensemble model called RF is also an effective model for author attribution. We use multilayer perceptron-based ANNs having one layer, two layers, and three layers, respectively. All the ANNs use fully connected layer and ReLU as the activation function and Adam as the optimizer. For our experiments, we used a Dell PowerEdge R710 (with two Intel Xeon X5660 CPUs and 128 GB RAM) running Ubuntu 18.10 operating system. The implementations of MNB, RF, and ANN are based on Scikit-Learn [Scikit-Learn 2019]; the implementation of SVM is based on LIBSVM [Chang and Lin 2011]; the implementation of AADF is based on Scikit-Learn and gcForest package [Zhou and Feng 2017]. For the AADF approaches, 3-fold cross-validation is used for producing class vector. The number of self-adaptive layer is automatically determined. The training set is split into two parts, including growing set and estimating set. We call the subsets generated from the training set as growing/estimating set to avoid confusion with the validation sets of 10-fold cross-validation. Then, we use the growing set to grow the cascade and the estimating set to estimate the prediction accuracy. The self-adaptive layer growing process is automatically terminated when the prediction accuracy on the estimating set is not improved. Then, the cascade is retrained based on the training set, which is merged by growing set and estimating set [Zhou and Feng 2017]. Accuracy is used to measure the results of different approaches. We calculate the average accuracy for each approach using the 10-fold cross-validation method with 4,410 samples for dataset 1 and 1,850 samples for dataset 2 .

To study the attribution performance of the different approaches on the original dimensionality of datasets, we run the experiments on dataset 1 and dataset 2 using the approaches without FS unit. Table 4 shows the results of different approaches without using FS unit. As we can see from the results, AADF clearly outperformed other approaches on the original dimensionality. After we investigate dataset 2, we found that the important reason why its accuracy is lower than dataset 1 is that many different active authors in Ed channel heavily perform similar or even same post flood that involves posting large numbers of posts with repetitive text in the channel, which increases the author attribution difficulty.

After studying the attribution performance of the approaches with original dimensionality, we evaluate the approaches with FS unit. We used ERT and RF and gradient boosting machine (GBM) 
Table 4. Comparisons of Accuracies of Tenfold Cross-validation of Different Approaches without FS

\begin{tabular}{cccccccccc}
\hline & \multicolumn{3}{c}{ Dataset 1 } & \multicolumn{4}{c}{ Dataset 2 } \\
MNB & RF & ANN & SVM & AADF & MNB & RF & ANN & SVM & AADF \\
\hline 69.14 & 93.45 & 92.95 & 93.38 & $\mathbf{9 5 . 2 4}$ & 63.68 & 90.70 & 91.35 & 90.27 & $\mathbf{9 2 . 1 1}$ \\
\hline
\end{tabular}

Table 5. Comparisons of Accuracies of 10-fold Cross-validation of Different Approaches with RF-FS

\begin{tabular}{lcccccccccc}
\hline MDI & \multicolumn{9}{c}{ Dataset 1 } & \multicolumn{5}{c}{ Dataset 2 } \\
& MNB & RF & ANN & SVM & AADF & MNB & RF & ANN & SVM & AADF \\
\hline $10^{-3}$ & 65.15 & 90.63 & 85.76 & 79.50 & $\mathbf{9 1 . 7 2}$ & 63.35 & 85.62 & 81.30 & 75.30 & $\mathbf{8 7 . 3 5}$ \\
$10^{-4}$ & 86.42 & 92.40 & 93.74 & 93.47 & $\mathbf{9 4 . 7 2}$ & 83.89 & 89.68 & 91.84 & 90.32 & $\mathbf{9 2 . 1 6}$ \\
$10^{-5}$ & 85.90 & 92.99 & 93.74 & 93.67 & $\mathbf{9 4 . 7 6}$ & 80.54 & 90.32 & 90.70 & 90.22 & $\mathbf{9 2 . 2 7}$ \\
$10^{-6}$ & 84.97 & 92.88 & 93.17 & 93.38 & $\mathbf{9 5 . 0 8}$ & 78.59 & 90.76 & 91.03 & 90.32 & $\mathbf{9 2 . 6 5}$ \\
$10^{-7}$ & 84.97 & 92.99 & 93.06 & 93.33 & $\mathbf{9 4 . 8 1}$ & 78.59 & 90.70 & 90.54 & 90.32 & $\mathbf{9 2 . 5 4}$ \\
\hline
\end{tabular}

to automatically select features. All of these can measure the importance score of each feature to learn the impact of features towards predicting the classes. To encourage diversity, which is crucial to ensemble construction [Zhou 2012], instead of using 500 trees and 650 trees for RF and ERT in the CFS layer, the tree ensemble models in the FS unit use 700 trees for RF and ERT. The importance value of a feature is calculated by mean decrease impurity (MDI) for FS using RF and ERT. A GBM framework called LightGBM developed by Microsoft [Ke et al. 2017] is used to achieve GBM. Gradient boosting decision trees (GBDT) and gradient-based one-side sampling (GOSS) are, respectively, used as the boosting type of the GBM to perform FS. GBDT is a tree-based ensemble learning method that is trained in sequence by fitting the residual errors. GOSS is an enhanced GBDT method using sampling that randomly ignores training samples with small gradients. Both of GBDT and GOSS FS units use 100 tree estimators. The author attribution performance has not improved further after adding more tree estimators for FS (and more trees also require more training time and increase the computational time). The GBM achieved by LightGBM can measure feature importance through the parameter called split, which is the number of times the feature is used in the GBM. For the RF and ERT-based FS, the feature importance values of all features are normalized and summed to 1 . As the GBM FS methods, LightGBM keeps the number of times the feature is used as the feature importance values (split) and does not normalize them. If a feature's importance value is smaller than the defined threshold, then this feature is discarded and not considered as important. A number of different thresholds of feature importance values are used to test the performance of different approaches. We set the minimum value of the threshold as 1 for GBM methods, and $10^{-7}$ for RF and ERT methods, to extract all the features with non-zero feature importance value when setting the thresholds as the minimum. For each round of 10 -fold cross-validation, FS measures the feature importance using training data of that round. Next, the training data with selected features (by FS) is used to train the learning model, and then the model is evaluated using the selected features on the validation set of that round. The average accuracy of 10-fold cross-validation is used to measure the performance of different approaches.

To study the performance of AADF with FS unit, we run experiments using AADF with FS unit and also compare AADF to different approaches with FS unit. Tables 5-8 present the accuracy of each approach with varying methods of FS by applying different feature importance thresholds. 
Table 6. Comparisons of Accuracies of 10-fold Cross-validation of Different Approaches with ERT-FS

\begin{tabular}{lcccccccccc}
\hline MDI & \multicolumn{9}{c}{ Dataset 1 } & \multicolumn{5}{c}{ Dataset 2 } \\
& MNB & RF & ANN & SVM & AADF & MNB & RF & ANN & SVM & AADF \\
\hline $10^{-3}$ & 43.42 & 88.34 & 82.74 & 67.21 & $\mathbf{8 9 . 3 7}$ & 41.89 & 78.38 & 74.86 & 55.24 & $\mathbf{7 9 . 6 2}$ \\
$10^{-4}$ & 87.73 & 92.88 & 93.56 & 93.61 & $\mathbf{9 4 . 2 9}$ & 84.97 & 90.32 & 91.62 & 90.49 & $\mathbf{9 2 . 2 7}$ \\
$10^{-5}$ & 85.71 & 92.99 & 92.88 & 93.40 & $\mathbf{9 4 . 6 9}$ & 80.49 & 90.49 & 91.30 & 90.32 & $\mathbf{9 2 . 1 1}$ \\
$10^{-6}$ & 84.10 & 93.13 & 93.29 & 93.31 & $\mathbf{9 5 . 4 2}$ & 78.38 & 90.38 & 90.92 & 90.27 & $\mathbf{9 2 . 7 0}$ \\
$10^{-7}$ & 84.06 & 93.27 & 93.36 & 93.36 & $\mathbf{9 5 . 2 2}$ & 78.32 & 90.59 & 90.86 & 90.27 & $\mathbf{9 2 . 5 4}$ \\
\hline
\end{tabular}

Table 7. Comparisons of Accuracies of 10-fold Cross-validation of Different Approaches with GBDT-FS

\begin{tabular}{lcccccccccc}
\hline Split & \multicolumn{9}{c}{ Dataset 1 } & \multicolumn{5}{c}{ Dataset 2 } \\
& MNB & RF & ANN & SVM & AADF & MNB & RF & ANN & SVM & AADF \\
\hline 100 & 74.01 & 89.43 & 86.60 & 82.88 & $\mathbf{9 0 . 4 5}$ & 83.14 & 89.46 & 91.14 & 89.14 & $\mathbf{9 2 . 1 1}$ \\
50 & 81.54 & 91.34 & 90.73 & 89.41 & $\mathbf{9 3 . 1 1}$ & 84.70 & 90.00 & 91.89 & 89.84 & $\mathbf{9 2 . 2 2}$ \\
10 & 87.39 & 92.31 & 93.47 & 93.40 & $\mathbf{9 4 . 5 8}$ & 83.95 & 89.84 & 90.81 & 90.16 & $\mathbf{9 2 . 0 5}$ \\
5 & 87.60 & 92.77 & 93.54 & 93.40 & $\mathbf{9 4 . 6 9}$ & 84.11 & 90.27 & 91.30 & 90.16 & $\mathbf{9 2 . 3 8}$ \\
1 & 86.83 & 92.86 & 93.76 & 93.42 & $\mathbf{9 4 . 7 6}$ & 83.51 & 90.38 & 91.68 & 90.43 & $\mathbf{9 2 . 5 4}$ \\
\hline
\end{tabular}

Table 8. Comparisons of Accuracies of 10-fold Cross-validation of Different Approaches with GOSS-FS

\begin{tabular}{lcccccccccc}
\hline Split & \multicolumn{9}{c}{ Dataset 1 } & \multicolumn{5}{c}{ Dataset 2 } \\
& MNB & RF & ANN & SVM & AADF & MNB & RF & ANN & SVM & AADF \\
\hline 100 & 70.95 & 89.52 & 85.28 & 81.54 & $\mathbf{8 9 . 8 9}$ & 83.03 & 88.65 & 91.03 & 88.49 & $\mathbf{9 2 . 0 0}$ \\
50 & 79.41 & 90.93 & 89.98 & 88.68 & $\mathbf{9 3 . 0 2}$ & 84.86 & 89.89 & 91.03 & 89.57 & $\mathbf{9 2 . 2 2}$ \\
10 & 86.96 & 92.59 & 93.36 & 93.42 & $\mathbf{9 4 . 5 1}$ & 84.81 & 90.11 & 92.00 & 90.43 & $\mathbf{9 2 . 2 7}$ \\
5 & 87.53 & 92.54 & 93.90 & 93.49 & $\mathbf{9 4 . 5 4}$ & 84.16 & 89.84 & 91.19 & 90.11 & $\mathbf{9 2 . 4 3}$ \\
1 & 86.67 & 92.88 & 93.67 & 93.58 & $\mathbf{9 4 . 4 0}$ & 83.41 & 90.43 & 91.51 & 90.32 & $\mathbf{9 2 . 3 2}$ \\
\hline
\end{tabular}

Tables 9-10 show the average number of selected features of each FS with different feature importance thresholds. We observed that the ERT-FS improved accuracy of AADF on dataset 1, and all the FS methods in this study are able to improve AADF accuracy on dataset 2 . This shows that our FS units are useful for AADF. Further, by using ERT-FS with the same MDI threshold $10^{-6}$, AADF attains the best accuracy results with $95.42 \%$ on dataset 1 and $92.70 \%$ on dataset 2 . The average numbers of selected features are 5011.9 on dataset 1 and 4667.8 on dataset 2, which enables AADF to achieve the best accuracy results through ERT-FS. Hence, AADF can use ERT-FS to significantly reduce the feature dimensionality, while its attribution performance is even improved. Among non-AADF approaches, the ANN achieves the best accuracy results with $93.90 \%$ on dataset 1 and $92.00 \%$ on dataset 2 . All the FS methods in this study improve the accuracy results of the MNB, SVM, and ANN approaches on both datasets. The RF-FS is able to improve the accuracy of $\mathrm{RF}$ on dataset 2. As we can see from the results, for all the FS units with all the feature importance thresholds used in the experiments of this study, AADF achieves better accuracy than other 
Table 9. Average Number of Selected Features of 10-fold Cross-validation of Different Feature Importance Threshold with RF-FS and ERT-FS

\begin{tabular}{ccccc}
\hline MDI & \multicolumn{2}{c}{ Dataset 1 } & \multicolumn{2}{c}{ Dataset 2 } \\
& RF-FS & ERT-FS & RF-FS & ERT-FS \\
\hline $10^{-3}$ & 166.6 & 86.5 & 141.3 & 49.1 \\
$10^{-4}$ & $2,291.8$ & $2,738.1$ & $2,323.7$ & $2,750.5$ \\
$10^{-5}$ & $4,054.1$ & 4,485 & $4,039.2$ & $4,159.2$ \\
$10^{-6}$ & 4,731 & $5,011.9$ & $4,528.6$ & $4,667.8$ \\
$10^{-7}$ & $4,736.7$ & $5,022.2$ & $4,528.8$ & $4,668.7$ \\
\hline
\end{tabular}

Table 10. Average Number of Selected Features of 10-fold Cross-validation of Different Feature Importance Threshold with GBDT-FS and GOSS-FS

\begin{tabular}{ccccc}
\hline Split & \multicolumn{2}{c}{ Dataset 1 } & \multicolumn{2}{c}{ Dataset 2 } \\
& GBDT-FS & GOSS-FS & GBDT-FS & GOSS-FS \\
\hline 100 & 292.5 & 249.7 & $1,317.3$ & $1,270.8$ \\
50 & 727 & 639.2 & $1,944.5$ & $1,889.8$ \\
10 & $2,208.9$ & $2,111.9$ & $2,612.6$ & $2,646.3$ \\
5 & $2,676.6$ & $2,628.4$ & $2,815.9$ & $2,932.2$ \\
1 & $3,649.3$ & $3,621.3$ & $3,190.5$ & $3,226.6$ \\
\hline
\end{tabular}

Table 11. Average Number of Selected Features Using RF-FS and ERT-FS with Threshold $10^{-6}$ and GBDT-FS with Threshold 1 and GOSS-FS with Threshold 5

\begin{tabular}{ccccccccccc}
\hline Feature Set & \multicolumn{3}{c}{ Dataset 1 } & \multicolumn{3}{c}{ Dataset 2 } \\
& Total & RF & ERT & GBDT & GOSS & Total & RF & ERT & GBDT & GOSS \\
\hline F1 & 94 & 92 & 92 & 92 & 91.9 & 94 & 92 & 92 & 91.1 & 90.2 \\
F2 & 1,500 & 1,500 & 1,500 & $1,496.6$ & $1,480.1$ & 1,500 & $1,498.5$ & 1,499 & $1,490.4$ & $1,487.6$ \\
F3 & 1,500 & $1,397.2$ & $1,487.3$ & 840.3 & 307.3 & 1,500 & $1,353.9$ & 1,396 & 768.9 & 640.6 \\
F4 & 543 & 484.2 & 505.5 & 418.2 & 275.7 & 543 & 492.3 & 498.9 & 391.3 & 364.9 \\
F5 & 6,702 & 815.5 & 953.5 & 454 & 212.7 & 6,702 & 809 & 894 & 249.6 & 172.7 \\
F6 & 46 & 22.7 & 28.1 & 14.4 & 8.6 & 46 & 24 & 24.9 & 11.6 & 10.4 \\
F7 & 37 & 16.2 & 17 & 13.9 & 10 & 37 & 15.8 & 15.3 & 7.5 & 5.9 \\
F8 & 48 & 48 & 48 & 46 & 46 & 48 & 48 & 48 & 46 & 46 \\
F9 & 52 & 52 & 52 & 52 & 52 & 52 & 52 & 52 & 52 & 52 \\
F10 & 441 & 303.2 & 328.5 & 221.9 & 144.1 & 185 & 143.1 & 147.7 & 82.1 & 61.9 \\
\hline
\end{tabular}

approaches. Hence, we conclude that AADF outperforms other approaches with FS units in this study.

To understand the contribution of different feature sets, we investigate the selected number of features and the percentage of usage of each feature set. Table 11 presents the average number of selected features and the total number of features of that feature set when performing 10 -fold cross-validation experiments. For dataset 1 and dataset 2, the cases of RF-FS and ERT-FS with a 


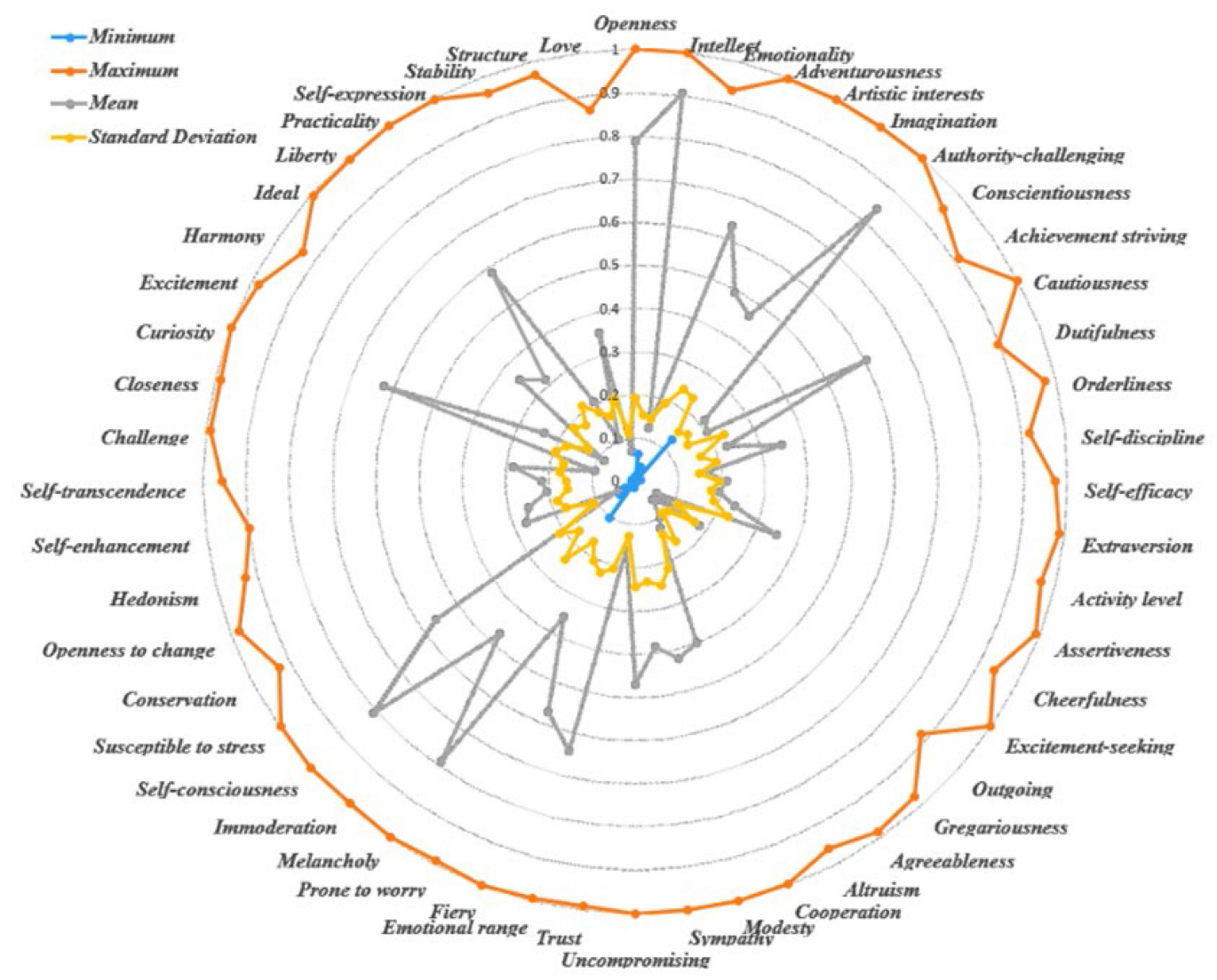

Fig. 5. Statistical results of personality insights features from 4,410 writing samples used in the experiments.

threshold of $10^{-6}$, GBDT-FS with a threshold of 1, and GOSS-FS with a threshold of 5 are selected to present, because AADF, respectively, achieved the best accuracy with them in RF-FS, ERT-FS, GBDT-FS, and GOSS-FS. As we can see, all the feature sets contribute features to the attribution model with the best performance in each FS unit. In addition, the feature sets of character n-grams (F2), word n-grams (F3), IRC stop-words (F4), and IRC cybersecurity knowledge and attitude (F5) contribute more features in each type FS method, since a lot of these features are included in their feature sets before FS. From the perspective of efficiency, we notice that IRC personality insights feature set (F9) achieved the best efficiency, because all of them are selected by the four different FS methods for both datasets. Figure 5 shows the statistic results of the F9 of 4,410 samples on dataset 1 before feature rescaling (min-max normalization), which can help researchers understand hacker behaviors through personality insights. The IRC activity behaviors (F8) and letters, numbers, special characters, and punctuations (F1) are also highly efficient feature sets. They have over 95\% usage by using different FS methods. The feature set of IRC public pair conversation network (F10) has good usage for RF-FS and ERT-FS on both datasets. For dataset 1, F10 has over $68 \%$ representation with ERT-FS and RF FS, whereas it attains over 50\% usage by GBDT-FS and over $30 \%$ usage by GOSS-FS. For dataset 2, F10 feature set has over 77\% representation by using ERT-FS and RF-FS, and it gets over $40 \%$ usage by GBDT-FS and over $30 \%$ usage by GOSS-FS. The explanation of the good usage of this feature set is that IRC users form their pair conversation networks based on friendship, common topics of interest, specialization, and location, which can help 
Table 12. The Top 10 Most Frequent Character N-grams and Word N-grams in Dataset 1

\begin{tabular}{|c|c|c|c|c|}
\hline \multirow[t]{2}{*}{$\overline{\text { Rank }}$} & \multicolumn{4}{|c|}{ Dataset 1} \\
\hline & Character 2-grams & Character 3-grams & Word 2-grams & Word 3-grams \\
\hline 1 & "e_" & “_th" & “it’s" & “i don’t \\
\hline 2 & “ t" & "the" & "don't" & "www.youtube.com" \\
\hline 3 & "t_" & "he_" & “i’m” & "youtube.com/watch" \\
\hline 4 & "s_" & "ing" & "in the" & "https://www.youtube" \\
\hline 5 & "th" & "ng_" & "of the" & "com/watch?v" \\
\hline 6 & “a” & "_to" & "if you" & “it’s not" \\
\hline 7 & "i" & "you" & "that's" & "you don't" \\
\hline 8 & "in" & “_yo" & “you can” & "a lot of" \\
\hline 9 & "he" & "to_" & "https://www" & “it’s a” \\
\hline 10 & "d_" & "_an" & "to be" & "I'm not" \\
\hline
\end{tabular}

Table 13. The Top 10 Most Frequent Character N-grams and Word N-grams in Dataset 2

\begin{tabular}{ccccc}
\hline Rank & & \multicolumn{2}{c}{ Dataset 2} & \\
& Character 2-grams & Character 3-grams & Word 2-grams & Word 3-grams \\
\hline 1 & "e_" & "th" & "it's" & "www.youtube.com" \\
2 & "_t" & "the" & "i'm" & if if if \\
3 & "t_" & "ing" & "don't" & "https://www.youtube" \\
4 & "s_" & "he_" & "in the" & "youtube.com/watch" \\
5 & "_a" & "ng_" & "is a" & "com/watch?v" \\
6 & "th" & "you" & "of the" & "i don't" \\
7 & "-i" & "_to" & "if you" & "friday it's \\
8 & "in" & "_yo" & "https://www" & "it's flood" \\
9 & "he" & "_an" & "i have" & "s flood friday" \\
10 & "d_" & "_a_" & "youtube.com" & "flood friday it" \\
\hline
\end{tabular}

to characterize the users. We also observed that the IRC abbreviation feature set (F6) and the IRC emoticons feature set (F7) have good usages for RF-FS and ERT-FS, while low usages for GBDTFS and GOSS-FS. F6 gets over 49\% usage using RF-FS and ERT-FS methods and over $18 \%$ usage by GBDT-FS and GOSS-FS methods for both datasets. F7 approaches over $40 \%$ representation by RF-FS and ERT-FS, and at least 15\% representation using GBDT-FS and GOSS-FS for both datasets. Due to the large number of n-grams features used by AADF, it is natural to ask which n-grams features most frequently appear. Thus, the top 10 most frequent n-grams of each $n$-grams feature set in our study are listed in Tables 12 and 13. It is interesting to note that the word 3-grams statistic reflects how frequently the similar or same flood posts appeared in dataset 2 . This fact increases the author attribution difficulty on dataset 2 .

\subsection{Computational Complexity Analysis}

The first priority of most machine learning approaches is to create models that maximize accuracy. As models with equivalent prediction performance, a secondary objective is usually to minimize time complexity. Therefore, we also provide time complexity analysis of the attribution model learning phase (without time complexity analysis of FS unit). The time complexity of building a tree in the worst case is, respectively, $O\left(\sqrt{d} n^{2} \log n\right)$ and $O\left(\sqrt{d} n^{2}\right)$ for a tree of RFs and a tree of 
Table 14. The Worst Time Complexity of Attribution Model Learning Phase

\begin{tabular}{lc}
\hline Approach & Time Complexity \\
\hline MNB & $O\left(d^{\prime} n\right)$ \\
ANN & $O\left(M I_{A N N} n d^{\prime} \prod_{H=1}^{b} a_{H}\right)$ \\
RF & $O\left(t \sqrt{d^{\prime}} n^{2} \log n\right)$ \\
SVM & $O\left(I_{S V M} n d^{\prime}\right)$ \\
AADF & $O\left(L w \sqrt{d^{\prime}} n^{2} \log n\right)$ \\
\hline
\end{tabular}

* for ANN, $a_{H}$ is number of neurons in hidden layer $H, b$ is total number of the hidden layers, and $I_{A N N}$ is number of iterations.

${ }^{* *} t$ is number of tree in RF approach.

${ }^{* * *} I_{S V M}$ is number of iteration of SVM.

ERTs, when given $d$ features and $n$ samples and setting the square root of the number of features are randomly drawn at each node [Louppe 2014]. Hence, in our approach, for a given training dataset $X=\left\{x_{i}, y_{i}\right\}\left(x_{i} \in \mathbb{R}^{d^{\prime}}, y_{i} \in Y,|Y|=M, i=1,2,3, \ldots, n\right)$, the time complexity for building a tree in the worst case is $O\left(\sqrt{d^{\prime}} n^{2} \log n\right)$ in the CFS, where $d^{\prime}$ is the total number of features after applying FS unit, $n$ is the total number of samples. Hence, the time complexity of processing each layer of CFS unit in the worst case is $O\left(w \sqrt{d^{\prime}} n^{2} \log n\right)$, where $w$ is the total number of trees built during the processing of each layer of CFS. Hence, the time complexity for the attribution model learning phase of AADF is $O\left(L w \sqrt{d^{\prime}} n^{2} \log n\right)$ in the worst case, where $L$ is the total number of generated layers during the growth of CFS. Please note that AADF adaptively decides $L$ in a datadependent way for different tasks. In our experiments, the values of $L$ are smaller than 4 in all the cases of dataset 1 and smaller than 5 in all the cases of dataset 2. The Scikit-Learn implementation of RF and ERT relies on NumPy vector operation and Cython for efficient numerical computing [Scikit-Learn 2019], which helps AADF to achieve scalability. Thus, AADF has acceptable computational performance and scalability. In addition, the training time can be further reduced if bootstrap samples are used.

We should note that the worst case of AADF happens in the very exceptional case when the splits are very unbalanced, which results in splits that move one sample in the first sub-tree and move all $n-1$ other samples in the second sub-tree during all the decision trees constructions. Due to the fact that the decision tree looks for splits that further decrease impurity, the frequency of balance partitions of the nodes samples should be higher than partitions that are very unbalanced. Therefore, trees are more likely to approach the case when node samples are partitioned into balanced subsets, than very unbalanced subsets resulting in the average time complexity of building one tree is, respectively, $\Theta\left(\sqrt{d^{\prime}} n \log ^{2} n\right)$ and $\Theta\left(\sqrt{d^{\prime}} n \log n\right)$ for RF and ERT [Louppe 2014]. Thus, the average time complexity of author attribution model learning phase of AADF is closer to $\Theta\left(L w \sqrt{d^{\prime}} n \log ^{2} n\right)$, resulting in good computational performance and scalability.

The worst time complexity of each approach is listed in Table 14. For the ANN, the time complexity is dominated by backpropagation. The worst time complexity of backpropagation is $O\left(M I_{A N N} n d^{\prime} \prod_{H=1}^{b} a_{H}\right)$ [Scikit-learn Neural Network Models 2019], which is generally quite high and may perform many iterations for convergence. With increasing the number of layers and neurons and iterations, the training time of ANN is increased significantly. For the SVM, the time complexity in the worst case is $O\left(I_{S V M} n d^{\prime}\right)$ [Chang and Lin 2011], and the optimization problem task of the dual problem of SVM can be efficiently solved by a sequential minimal 
Table 15. This Research's Impact on Other Areas of IRC Authorship Analysis

\begin{tabular}{cll}
\hline \multicolumn{1}{c}{ Area } & \multicolumn{1}{c}{ Description of the Area } & \multicolumn{1}{c}{ Impact } \\
\hline IRC author verification & $\begin{array}{l}\text { Verifying whether the unknown texts } \\
\text { are written by a particular IRC user } \\
\text { Grouping unlabeled texts that are } \\
\text { written by the same IRC user }\end{array}$ & $\begin{array}{l}\text { Feature extraction methods F1-F10; } \\
\text { AADF-based author verification model }\end{array}$ \\
Feature extraction methods F1-F9 \\
$\begin{array}{l}\text { Analyzing IRC user profiling aspects } \\
\text { including age, native language, gender, } \\
\text { or personality }\end{array}$ & $\begin{array}{l}\text { Personality insight analysis; } \\
\text { AADF-based author profiling } \\
\text { classification model }\end{array}$ \\
\hline
\end{tabular}

optimization-type decomposition method. It is empirically known that the number of iterations of performing decomposition method may be higher than linear to the number of training samples, resulting in considerable training time for huge datasets [Chang and Lin 2011]. In our cases, the time complexity of SVM is lower than AADF. The MNB approach has a time complexity of $O\left(d^{\prime} n\right)$ [Zheng and Geoffrey 2005], which is the best among all the approaches in this study. MNB is simple and has an efficient computational nature, due to the independence assumption of the features, though this assumption is always violated.

\section{DISCUSSION AND FUTURE WORKS}

Although we demonstrated that our approach works well for the author attribution in IRC channels, this study can also provide direction for authorship identification tasks on other social media platforms. In the feature extraction phase, the feature sets F1, F2, F3, F4, F5, and F9 can be directly applied to forensic investigations of other platforms due to their generalization. The remaining feature sets (i.e., F6, F7, F8, and F10) provide a high-level concept of feature extraction methods for other social media applications, though platform-specific feature extraction might be required. The AADF ensemble model achieved the best performance in all cases in our experiments in the same setting. Hence, we believe that our ensemble learning-based model would perform well for author attribution tasks across multiple social media platforms, because ensemble classifier usually provides better classification results than one-classifier. The AADF can be viewed as an ensemble of ensembles that leverages almost all strategies for diversity enhancement. Moreover, this study can significantly offer value to other areas of IRC authorship analysis, such as author verification [Iqbal et al. 2010; Shao et al. 2019a], author clustering [Tschuggnall et al. 2017; Shao et al. 2019b], and author profiling [Estival et al. 2007]. For IRC author verification, all the feature extractors can be directly applied. For the learning model, one way to deal with IRC author verification problem is to formulate it as a binary classification problem. The AADF is able to transform from a multi-class classification model to a binary classification model by using two-class training data. For IRC author clustering, all the feature extraction methods in this study still can be leveraged except the F10 feature set requiring specific candidate information. As IRC author profiling, using the personality insight feature extraction method is a straightforward way to analyze personality. The RF, SVM, and MNB are widely utilized for author profiling [Kavuri and Kavitha 2020]. Hence, our ensemble of ensembles-based authorship analysis approach provides an opportunity to improve the accuracy due to its best performance in this study, which also falls in the area of the high-dimensional text classification problem. We summarize the extension of our work in terms of other IRC authorship analysis areas in Table 15.

It should be noted that author identification approach (including our approach) is unlikely to perform very well if suspects intentionally disseminate conversations for contaminating statistics or if an account is used by multiple users. Like most machine learning models, our tree-based 
ensemble learning model relies on the i.i.d. assumption. Careful cybercriminals could disguise their writing to ensure that writing samples violate the i.i.d assumption, thus reducing the possibility of identification. An interesting study [Brennan et al. 2012] developed three disguised writing methods for circumventing authorship recognition: obfuscation, imitation, and machine translation. Their results showed that manually disguised writing methods, including obfuscation and imitation, work well, whereas automated translation is ineffective. In IRC, one promising way to improve the success rate against disguised writing methods is to collect long-term data of suspects, since successful circumventing authorship analysis is limited to manual methods that require hand-crafted manipulation [McDonald et al. 2012] or semi-auto methods that provide possible modification suggestion [Castro-Castro et al. 2017], and using these methods consistently is even hard for sophisticated hackers. This emphasizes the importance of IRC data collection and how our autonomic IRC bot framework should be deployed. In addition, the adoption of nontraditional feature sets, such as personality insight, may reveal the adversarial IRC authors who attempt to circumvent author attribution system through contaminating traditional writing features documented in the literature. ANNs achieved good author attribution performance in this study. However, ANNs are susceptible to adversarial examples such as inputs that are similar to a correctly classified input but are misclassified [Chen et al. 2019]. This has led to extensive studies on the use of maliciously crafted adversarial examples to attack ANNs, providing another possible option for attacking ANN-based author attribution models. When creating adversarial examples, most methods compute the gradient descent of the ANNs [Chen et al. 2019]. Our approach is more robust than ANNs under such attacks, because CFS is based on non-differentiable module without performing gradient descent-based adjustment. Thus, common gradient descent-based attacks cannot be used to attack our approach.

In addition, we have evaluated our approach in a closed environment using batch data (i.e., data was collected and then we applied author identification analysis offline). One important future issue is performing IRC author attribution on data streams for which the environment is open and may change arbitrarily or even adversarially. In the streaming IRC author attribution environment, the joint distribution between IRC writing features and the target suspects changes. This problem is referred to as concept drift [Gama et al. 2014]. The classification performance of AADF will drop if we ignore the distribution change, which is not desirable for IRC author attribution. Adaptive random forest (ARF) is an adaptation of the classical $\mathrm{RF}$ that can deal with evolving data streams [Gomes et al. 2017]. ARF combines batch algorithm traits with dynamic update methods that can cope with various types of concept drift without complicated optimization. Therefore, one possible solution is to use ARF as the base tree ensemble model in AADF.

\section{CONCLUSION}

The anonymity of Internet services, especially in social media platforms, provides users with freedom. However, such anonymity can be exploited for underground cyber-criminal activity. Thus, it is critically important to develop methods to identify anonymous users involved in cybercrimes. To address this social media forensics challenge, we presented a new ensemble of ensembles-based IRC author attribution forensic technique to accurately identify candidate authors from a large number of candidate sets. In our approach, we use the first author attribution version of deep forest to adaptively learn novel IRC writing features to identify IRC users. Unlike previous author attribution research in IRC who suffered from the problem of low accuracy in heterogeneous topic channels with many candidates, our approach achieved high accuracy with a limited number of samples. Also, we developed an autonomic IRC monitoring environment that can effectively collect and classify messages in real-time from cybersecurity channels ignored by previous IRC author identification works. 


\section{A APPENDICES}

In the Appendices section, we list the used IRC abbreviations and IRC emoticons in this study.

\section{A.1 IRC Emoticons}

\author{
:) (smile) \\ ;-) (wink with a nose) \\ $:^{*}$ (kisses) \\ : ((frown) \\ $:-<$ (forlorn) \\ :-! (foot in mouth - oops!) \\ [:-| (frankenstein) \\ $: /($ wry) \\ (o_o) (shocked) \\ \&:-) (a person with curly hair) \\ :-> (smile with definite sarcasm) \\ $\sim:-($ steaming mad) \\ $<:-)$ (asking dumb question)
}

\section{A.2 IRC Abbreviation}

afk (away from the keyboard)

bbs (be back shortly)

bbiaf (be back in a few)

ctc (care to chat?)

focl (falling off chair laughing)

$<\mathrm{g}>$ (Grin)

imo (in my opinion)

irl (in real life)

lts (laughing to self)

ly (love you)

$\mathrm{n} / \mathrm{p}$ (no problem)

ppl (people)

rofl (rolling on floor laughing)

tafn (that's all for now)

ttys (talk (type) to you soon)

WYSIWYG (what you see is what you get)

$:-)$ (smile with a nose)
$: \mathrm{D}$ (very wide grin)
$:^{* *}$ : (returning kiss)
$>$ :((angry frown)
$: \mathrm{x}$ (my lips are sealed)
$\mathrm{P}^{*}$ (french kiss)
$\wedge 5$ (High Five!)
$=:-\mathrm{O}$ (Oh, I'm scared!)
$\sim \backslash 0 / \sim$ (waving hello or goodbye)
\#:-) (from a person with matted hair)
$\left.{ }^{*}<\mid:-\right)$ (santa claus or clown)
$\backslash /$ (empty glass)

;) (wink)

:P (sticking out tongue)

:I (hmmm...)

:[(real downer)

:-o (oh, no!)

O:) (angel)

$=-\mathrm{O}$ (the enterprise)

(@_@) (stunned)

:-))) (big happy smile)

:> (smile with a sarcasm)

${ }^{\wedge}{ }^{\wedge}=($ a cat smiley $)$

$\sim$ (full glass)

\section{REFERENCES}

F. Amuchi, A. Al-Nemrat, M. Alazab, and R. Layton. 2012. Identifying cyber predators through forensic authorship analysis of chat logs. In Proceedings of the IEEE 3rd Cybercrime and Trustworthy Computing Workshop 28-37.

A. Abbasi and H. Chen. 2005. Applying authorship analysis to extremist-group web forum messages. IEEE Intell. Syst. 20, 5 (2005), 67-75

A. Abbasi and H. Chen. 2008. Writeprints: A stylometric approach to identity-level identification and similarity detection in cyberspace. ACM Trans. Inf. Syst. 26, 2 (2008), 7.

M. H. Altakrori, F. Iqbal, B. Fung, S. H. Ding, and A. Tubaishat. 2018. Arabic authorship attribution: An extensive study on Twitter posts. ACM Trans. Asian Low-resour. Lang. Inf. Proc. 18, 1 (2018), 5.

S. Alrabaee, N. Saleem, S. Preda, L. Wang, and M. Debbabi. 2014. Oba2: An onion approach to binary code authorship attribution. Dig. Investig. 11 (2014), S94-S103.

L. Breiman. 2001. Random forests. Machine Learn. 45, 1 (2001), 5-32. 
G. Baron. 2016. Comparison of cross-validation and test sets approaches to evaluation of classifiers in authorship attribution domain. In Proceedings of the International Symposium on Computer and Information Sciences. Springer, Cham, 81-89.

M. Brennan, S. Afroz, and R. Greenstadt. 2012. Adversarial stylometry: Circumventing authorship recognition to preserve privacy and anonymity. ACM Trans. Inf. Syst. Sec. 15, 3 (2012), 1-22.

V. Benjamin and H. Chen. 2014. Time-to-event modeling for predicting hacker IRC community participant trajectory. In Proceedings of the IEEE International Conference on Intelligence and Security Informatics (ISI'14). 25-32.

V. Benjamin and H. Chen. 2015. Developing understanding of hacker language through the use of lexical semantics. In Proceedings of the IEEE International Conference on Intelligence and Security Informatics (ISI'15). 79-84.

V. Benjamin, B. Zhang, J. F. Nunamaker Jr, and H. Chen. 2016. Examining hacker participation length in cybercriminal internet-relay-chat communities. F. Manag. Inf. Syst. 33, 2 (2016), 482-510.

H. Baayen, H. Halteren, A. Neijt, and F. Tweedie. 2012. An experiment in authorship attribution. In Proceedings of the International Conference on Statistical Analysis of Textual Data. 29-37.

A. Białecki, R. Muir, G. Ingersoll, and Lucid Imagination. 2012. Apache lucene 4. In Proceedings of the SIGIR Workshop on Open Source Information Retrieval. 17.

J. Bernard, S. Shao, C. Tunc, H. Kheddouci, and S. Hariri. 2018. Quasi-cliques analysis for IRC channel thread detection. In Proceedings of 7th International Conference on Complex Networks and their Applications. 578-589.

D. Castro-Castro, R. O. Bueno, and R. Muñoz. 2017. Author masking by sentence transformation. In Proceedings of the Conference and Labs of the Evaluation Forum (CLEF'17) (Working Notes).

C.-C. Chang and C.-J. Lin. 2011. LIBSVM: A library for support vector machines. ACM Trans. Intell. Syst. Technol. 2, 3 (2011), $1-27$.

D. A. Cobb-Clark and S. Schurer. 2012. The stability of big-five personality traits. Econ. Lett. 115, 1 (2012), 11-15

H. Chen, H. Zhang, D. Boning, and C.-J. Hsieh. 2019. Robust decision trees against adversarial examples. In Proceedings of the International Conference on Machine Learning.

P. Y. Du and N. Zhang. 2018. Hacker web forum collection: IRC conversation for channel. University of Arizona Artificial Intelligence Lab, AZSecure-data. Retrieved from http://www.azsecure-data.org/.

D. Estival, T. Gaustad, S. B. Pham, W. Radford, and B. Hutchinson. 2007. Author profiling for English emails. In Proceedings of the 10th Conference of the Pacific Association for Computational Linguistics. 263-272.

H. M. Gomes, A. Bifet, J. Read, J. Barddal, F. Enembreck, B. Pfharinger, H. Geoff, and T. Abdessalem. 2017. Adaptive random forests for evolving data stream classification. Mach. Learn. 106 (9-10) (2017), 1469-1495.

P. Geurts, D. Ernst, and L. Wehenkel. 2006. Extremely randomized trees. Mach. Learn. 63, 1 (2006), 3-42.

J. Gama, I. Žliobaitè, A. Bifet, M. Pechenizkiy, and Abdelhamid Bouchachia. 2014. A survey on concept drift adaptation. ACM Comput. Surv. 46, 4 (2014), 1-37.

G. Hirst and O. Feiguina. 2007. Bigrams of syntactic labels for authorship discrimination of short texts. Liter. Ling. Comput. 22, 4 (2007), 405-417.

G. Inches, M. Harvey, and F. Crestani. 2013. Finding participants in a chat: Authorship attribution for conversational documents. In Proceedings of the International Conference on Social Computing (SocialCom'13). 272-279.

F. Iqbal, L. A. Khan, B. C. M. Fung, and M. Debbabi. 2010. E-mail authorship verification for forensic investigation. In Proceedings of the ACM Symposium on Applied Computing. 1591-1598.

IBM Watson Assistant. 2018. IBM Watson Assistant service. Retrieved from https:/www.ibm.com/cloud/watson-assistant/.

IBM Watson Personality Insights. 2018. IBM Watson Personality Insights service. Retrieved from https://console.bluemix. net/docs/services/personality-insights.

Imbalanced-learn API. 2019. RandomUnderSampler. Retrieved from https://imbalanced-learn.readthedocs.io/en/stable/ generated/imblearn.under_sampling.RandomUnderSampler.html.

IRC Abbreviations. 2018. Introduction to IRC Abbreviations. Retrieved from http://www.ircbeginner.com/ircinfo/ abbreviations.html.

IRC Emoticons. 2018. Introduction to IRC Emoticons. Retrieved from http://www.ircbeginner.com/ircinfo/emoticons.html.

B. Kratzwald and S. Feuerriegel. 2019. Putting question-answering systems into practice: Transfer learning for efficient domain customization. ACM Trans. Manag. Inf. Syst. 9, 4 (2019), 1-20.

K. Kavuri and M. Kavitha. 2020. A stylistic features based approach for author profiling. In Recent Trends in Communication and Intelligent Systems. Springer, Singapore, 185-193.

G. Ke, Q. Meng, T. Finley, T. Wang, W. Chen, W. Ma, and T. Y. Liu. 2017. Lightgbm: A highly efficient gradient boosting decision tree. In Proceedings of the Conference on Advances in Neural Information Processing Systems. 3146-3154.

G. Louppe. 2014. Understanding Random Forests: From Theory to Practice. Dissertation. University of Liege, Belgium.

K. Luyckx and W. Daelemans. 2008. Authorship attribution and verification with many authors and limited data. In Proceedings of the 22nd International Conference on Computational Linguistics (COLING'08). 513-520.

S. H. Li, Y. C. Kao, Z. C. Zhang, Y. P. Chuang, and D. C. Yen. 2015. A network behavior-based botnet detection mechanism using PSO and K-means. ACM Trans. Manag. Inf. Syst. 6, 1 (2015), 1-30. 
R. Y. Lau, S. Y. Liao, R. C. W. Kwok, K. Xu, Y. Xia, and Y. Li. 2012. Text mining and probabilistic language modeling for online review spam detection. ACM Trans. Manag. Inf. Syst. 2, 4 (2012), 1-30.

R. Layton, S. McCombie, and P. Watters. 2012. Authorship attribution of IRC messages using inverse author frequency. In Proceedings of the 3rd IEEE Cybercrime and Trustworthy Computing Workshop. 7-13.

R. Layton, P. Watters, and R. Dazeley. 2013. Local n-grams for author identification. Notebook for PAN at CLEF 2013. In Proceedings of the Conference and Labs of the Evaluation Forum and Workshop (CLEF'13).

X. Liu, J. Wu, and Z. Zhou. 2009. Exploratory undersampling for class-imbalance learning. IEEE Trans. Syst., Man, Cybernetics, Part B (Cyber.) 39, 2 (2009).

A. McDonald, S. Afroz, A. Caliskan, A. Stolerman, and R. Greenstadt. 2012. Use fewer instances of the letter "i": Toward writing style anonymization. In Proceedings of the International Symposium on Privacy Enhancing Technologies Symposium. Springer, Berlin, 299-318.

C. D. Manning, P. Raghavan, and H. Schuetze. 2008. Introduction to Information Retrieval. Cambridge University Press, 234-265

S. Maruf, K. Javed, and H. A. Babri. 2016. Improving text classification performance with random forests-based feature selection. Arab. F. Sci. Eng. 41, 3 (2016), 951-964.

$\mathrm{X}$. Mu, K. Ting, and Z. Zhou. 2017. Classification under streaming emerging new classes: A solution using completelyrandom trees. IEEE Trans. Knowl. Data Eng. 29, 8 (2017), 1605-1618.

R. Mukras, N. Wiratunga, R. Lothian, S. Chakraborti, and D. Harper. 2007. Information gain feature selection for ordinal text classification using probability re-distribution. In Proceedings of the Textlink Workshop at IJCAI.

A. Rajaraman and J. D. Ullman. 2011. Mining of Massive Datasets. Cambridge University Press.

E. Stamatatos. 2009. A survey of modern authorship attribution methods. J. Amer. Soc. Inf. Sci. Technol. 60, 3 (2009), 538-556.

M. Sauter. 2014. The Coming Swarm: DDOS Actions, Hacktivism, and Civil Disobedience on the Internet. Bloomsbury Academic, New York, NY.

NIST. 2018. Glossary of key information security terms. Retrieved from https://csrc.nist.gov/glossary.

Scikit-Learn API. 2019. Scikit-learn: machine learning in Python. Retrieved from https://scikit-learn.org/stable/.

Scikit-Learn MinMaxScaler. 2019. Sklearn Preprocessing MinMaxScaler. Retrieved from https://scikit-learn.org/stable/ modules/generated/sklearn.preprocessing.MinMaxScaler.html.

Scikit-Learn Neural Network Models. 2019. Neural Network Models (Supervised). Retrieved from https://scikit-learn.org/ stable/modules/neural_networks_supervised.html.

S. Samtani and H. Chen. 2016. Using social network analysis to identify key hackers for keylogging tools in hacker forums. In Proceedings of the IEEE Conference on Intelligence and Security Informatics (ISI'16). 319-321.

S. Samtani, R. Chinn, H. Chen, and J. F. Nunamaker Jr. 2017. Exploring emerging hacker assets and key hackers for proactive cyber threat intelligence. F. Mana. Inf. Syst. 34, 4 (2017), 1023-1053.

S. Samtani, K. Chinn, C. Larson, and H. Chen. 2016. AZSecure hacker assets portal: Cyber threat intelligence and malware analysis. In Proceedings of the IEEE Conference on Intelligence and Security Informatics (ISI'16). 19-24.

S. Segarra, M. Eisen, and A. Ribeiro. 2015. Authorship attribution through function word adjacency networks. IEEE Trans. Sig. Proc. 63, 20 (2015), 5464-5478.

E. Stamatatos, N. Fakotakis, and G. Kokkinakis. 2000. Automatic text categorization in terms of genre and author. Comput. Ling. 26, 4 (2000), 471-495

T. Solorio, S. Pillay, S. Raghavan, and M. Montes-Gomez. 2011. Modality specific meta features for authorship attribution in web forum posts. In Proceedings of the International foint Conference on Natural Language Processing. 156-164.

R. Socher, A. Perelygin, J. Wu, J. Chuang, C. D. Manning, A. Ng, and C. Potts. 2013. Recursive deep models for semantic compositionality over a sentiment treebank. In Proceedings of the Conference on Empirical Methods in Natural Language Processing. 1631-1642.

S. Shao, C. Tunc, A. Al-Shawi, and S. Hariri. 2018. Autonomic author identification in internet relay chat (IRC). In Proceedings of the IEEE/ACS 15th International Conference on Computer Systems and Applications (AICCSA'18). 1-8.

S. Shao, C. Tunc, A. Al-Shawi, and S. Hariri. 2019a. One-class classification with deep autoencoder neural networks for author verification in internet relay chat. In Proceedings of IEEE/ACS 16th International Conference on Computer Systems and Applications (AICCSA'19). 1-8.

S. Shao, C. Tunc, A. Al-Shawi, and S. Hariri. 2019b. Automated Twitter author clustering with unsupervised learning for social media forensics. In Proceedings of the IEEE/ACS 16th International Conference on Computer Systems and Applications (AICCSA'19). 1-8.

R. Sedgewick and K. Wayne. 2018. Algorithms fourth edition: Algorithms, 3.5 searching applications, stop words. Retrieved from https://algs4.cs.princeton.edu/35applications/stopwords.txt.

The Register Security. 2012. UK cops: How we sniffed out convicted anonops admin "nerdo." Retrieved from https://www. theregister.co.uk/2012/12/14/uk_anon_investigation/. 
R. H. R. Tan and F. S. Tsai. 2010. Authorship identification for online text. In Proceedings of the International Conference on Cyberworlds (CW'10). 155-162.

M. Tschuggnall, E. Stamatatos, B. Verhoeven, W. Daelemans, G. Specht, B. Stein, and M. Potthast. 2017. Overview of the author identification task at PAN-2017: Style breach detection and author clustering. In Proceedings of the Conference and Labs of the Evaluation Forum and Workshop (CLEF'17).

K. Williams, J. Wu, and C. Lee Giles. 2014. Simseerx: A similar document search engine. In Proceedings of the ACM Symposium on Document Engineering. 143-146.

W. Wu, J. Zhou, Y. Xiang, and L. Xu. 2013. How to achieve non-repudiation of origin with privacy protection in cloud computing. F. Comput. Syst. Sci. 79, 8 (2013), 1200-1213.

Z. Zhou. 2012. Ensemble Methods: Foundations and Algorithms. CRC, Boca Raton, FL.

Z. Zhou and J. Feng. 2017. Deep forest: Towards an alternative to deep neural networks. In Proceedings of the 26th International foint Conference on Artificial Intelligence. 3553-3559.

F. Zheng and I. Geoffrey. 2005. A comparative study of semi-naive Bayes methods in classification learning. In Proceedings of the 4th Australasian Data Mining Workshop. 141-156.

R. Zheng, J. Li, H. Chen, and Z. Huang. 2006. A framework for authorship identification of online messages: Writing-style features and classification techniques. F. Amer. Soc. Inf. Sci. Technol. 57, 3 (2006), 378-393.

Received November 2019; revised June 2020; accepted June 2020 